\title{
Learning Infant Brain Developmental Connectivity for Cognitive Score Prediction ${ }^{\star}$
}

\author{
$\mathrm{Yu} \mathrm{Li}^{1 \dagger}$, Jiale Cheng ${ }^{1,2 \dagger}$, Xin Zhang ${ }^{1}$, Ruiyan Fang ${ }^{1}$, Lufan Liao ${ }^{1}$, Xinyao \\ Ding ${ }^{1}$, Hao $\mathrm{Ni}^{3}$, Xiangmin $\mathrm{Xu}^{1}$, Zhengwang $\mathrm{Wu}^{2}$, Dan $\mathrm{Hu}^{2}$, Weili $\mathrm{Lin}^{2}$, Li \\ Wang $^{2}$, John Gilmore ${ }^{2}$, and Gang $\mathrm{Li}^{2}$ \\ 1 South China University of Technology, Guangzhou, China \\ eexinzhang@scut.edu.cn \\ 2 University of North Carolina at Chapel Hill, Chapel Hill, USA \\ 3 University College London, London, UK
}

\begin{abstract}
During infancy, the human brain develops rapidly in terms of structure, function and cognition. The tight connection between cognitive skills and brain morphology motivates us to focus on individual level cognitive score prediction using longitudinal structural MRI data. In the early postnatal stage, the massive brain region connections contain some intrinsic topologies, such as small-worldness and modular organization. Accordingly, graph convolutional networks can be used to incorporate different region combinations to predict the infant cognitive scores. Nevertheless, the definition of the brain region connectivity remains a problem. In this work, we propose a crafted layer, the Inter-region Connectivity Module (ICM), to effectively build brain region connections in a data-driven manner. To further leverage the critical cues hidden in the development patterns, we choose path signature as the sequential data descriptor to extract the essential dynamic information of the region-wise growth trajectories. With these region-wise developmental features and the inter-region connectivity, a novel Cortical Developmental Connectivity Network (CDC-Net) is built. Experiments on a longitudinal infant dataset within 3 time points and hundreds of subjects show our superior performance, outperforming classical machine learning based methods and deep learning based algorithms.
\end{abstract}

Keywords: Infant Cognition Prediction - Brain Region Connectivity · Longitudinal Analysis.

\section{Introduction}

Infancy is an immense period to shape individuals' cognitive abilities [9]. Therefore, building the direct quantitative relationship between the longitudinal cor-

\footnotetext{
* This work is supported by Science and Technology Program of Guangzhou (2018-1002-SF-0561) and Natural Science Foundation of Guangdong Province (2018A030313295) to X Z.

$\dagger$ Equal contribution.
} 
tical structure and the cognitive scales is significant for us to better understand early brain development and related brain disorders. In this study, we aim to take longitudinal brain structural MRI scans as input and predict Mullen scales of early learning for each individual infant. Due to the challenges caused by the small sample size problem and the dynamic brain characteristics of infants, there are very few studies involving cognitive prediction $[1,23]$.

Learning a set of compact representations, which effectively capture the spatial and temporal cortical developmental patterns, is one of the most important techniques to deal with the small sample size problem. However, existing methods usually simply flatten brain morphological feature vectors at every time point into a vector $[1,23,24]$, which obviously neglect the potential connectivity among brain regions. It has been revealed that the massive brain region connections form elegant topologies, such as small-worldness and modular organization, which can be probed using the graph theoretical modeling method [25]. Hence, in recent works of functional MRI analysis [7,19], researchers build brain connectivity by Pearson's correlation. This kind of brain connectivity may also be constructed from structural MRI data and provide rich and useful information $[18,6]$.

To explore the hidden dynamic information in growth trajectories, previous works utilize a set of learnable matrices to fuse the structural information of brain regions and get the predicted cognitive scales $[1,23]$. However, as brain regions develop hetergeneously during infancy [16], it may be beneficial to get a more effective temporal feature representation by designing a region-wise dynamic dependency descriptor for the developmental trajectory of each region. Path signature is a graded sequence of statistics to characterize streamed data. It has been applied to acquire effective features of the path $[13,17,14,24]$, and might be helpful to extract the developmental information in brain regions.

In this work, our contributions are in three aspects. 1) We innovatively propose a Inter-region Connectivity Module (ICM) to adaptively connect brain region pairs for constructing brain region developmental connectivity. 2) Path signature is used to extract the developmental features to describe the growth trajectory of each brain region. 3) Taking the learned connectivity as an adjacent matrix and the developmental path signature feature as the node feature, a Graph Convolutional Network (GCN) based predictor is leveraged to predict the cognitive scores. The whole structure is called the Cortical Developmental Connectivity Network (CDC-Net). Extensive experiments showed that our method achieves the state-of-the-art performance among various baselines.

\section{Dataset and Feature Extraction}

Longitudinal T1w and T2w brain MR images from 110 subjects at 0,1 and 2 years of age were acquired. The T1w imaging parameters were $\mathrm{TR} / \mathrm{TE}=$ $1900 / 4.38 \mathrm{~ms}$, Flip Angle $=7^{\circ}$, and isotropic $1 \mathrm{~mm}$ resolution; and the $\mathrm{T} 2 \mathrm{w}$ imaging parameters were $\mathrm{TR} / \mathrm{TE}=7380 / 119 \mathrm{~ms}$, Flip Angle $=150^{\circ}$, and resolution $=1.25 \times 1.25 \times 1.95 \mathrm{~mm}^{3}$. All images were processed by an infant-tailored 


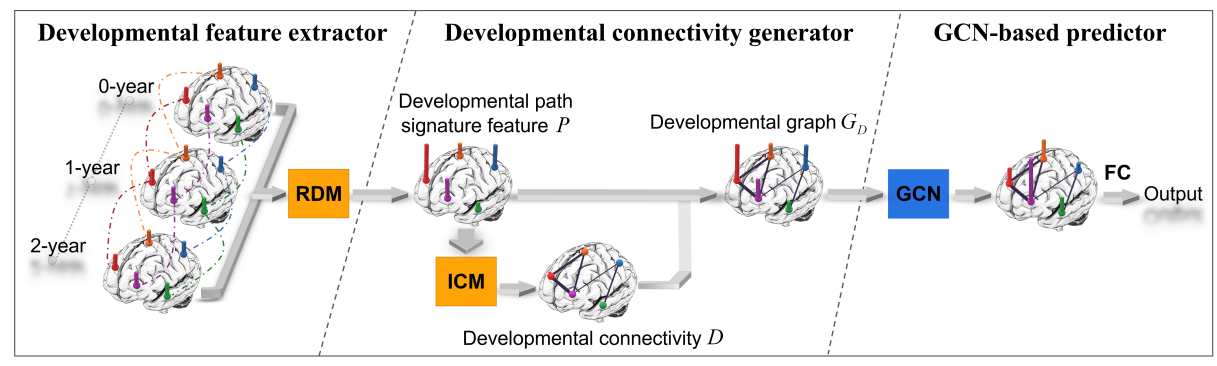

Fig. 1. Flowchart of our proposed method, which consists of three parts, including the developmental feature extractor, the developmental connectivity generator and a GCNbased predictor. Two modules: Region-wise Development Module (RDM) and Interregion Connectivity Module (ICM), are proposed to extract developmental feature $P$ and developmental connectivity $D$.

public computational pipeline ${ }^{\dagger}$. For each hemisphere, inner and outer cortical surfaces were reconstructed [15] and 4 morphological features for each vertex were computed, including the cortical thickness, surface area, average convexity, and mean curvature. Then, we mapped the inner cortical surface onto a sphere using FreeSurfer [5] and aligned the spherical surface onto the 4D Infant Cortical Surface Atlas ${ }^{\dagger}$ to propagate the Desikan [4] parcellation with 70 regions from the atlas onto each indiviudal surface. Finally, for each region, a 4-dimensional feature, i.e., the average cortical thickness, total surface area, average absolute convexity, and average absolute mean curvature were computed. Five Mullen cognitive scores of early learning are estimated for each participant at 2 years of age, i.e., Visual Receptive Scale (VRS), Fine Motor Scale (FMS), Receptive Language Scale (RLS), Expressive Language Scale (ELS) and Early Learning Composite (ELC).

\section{CDC-Net}

As illustrated in Fig. 1, our network consists of the development feature extractor, developmental connectivity generator and GCN-based predictor. Regionwise Development Module (RDM) is proposed to extract developmental features $P$ of the longitudinal brain structural data. We select the developmental path signature features, which combines path signature theory and deep learning framework to generate developmental path signature feature for each brain region. Then, an Inter-region Connectivity Module (ICM) is proposed to learn the relationship between brain region pairs, and generate a developmental connectivity matrix $D$.

By considering brain regions as nodes, the developmental feature $P$ can be seen as node features, and the developmental connectivity matrix $D$ is actually

\footnotetext{
$\dagger$ http://www.ibeat.cloud

$\dagger$ https://www.nitrc.org/projects/infantsurfatlas/
} 
an adjacent matrix of graph. By combining $P$ and $D$, we acquire a complete developmental graph $G_{D}=(P, D)$ to represent the developmental brain network. Finally, a GCN-based score predictor which contains one 2-layer GCN and one fully connected (FC) layer is used to predict the cognitive score.

\subsection{Region-wise Development Module (RDM)}

The region-wise development module is designed to extract the developmental features for each brain region, which is critical to build a meaningful developmental connectivity matrix $D$. We can summarize the function of RDM as follows:

$$
P_{i}=f_{R D M}\left(X_{i}\right)
$$

where $X \in \mathbb{R}^{70 \times 3 \times 4}$ represents our whole brain structural MRI data, $X_{i} \in \mathbb{R}^{3 \times 4}$ is longitudinal features of the $i^{\text {th }}$ region, and $P_{i} \in \mathbb{R}^{32}$ means the developmental feature of region $i$. The function $f_{R D M}(\cdot)$ can be any sequential feature extractor, which takes in the longitudinal feature of a brain region and returns its developmental features. In this work, we specifically introduce the path signature method as one of its instantiations.

Path Signature Preliminary. Suppose $\chi:[a, b] \rightarrow \mathbb{R}^{d}$ is a $d$-dimensional path defined on the time interval $[a, b]$. Conventionally, we regard the sequential data such as the region-wise growth trajectory, has a natural path-like structure. For any $t \in[a, b], \chi_{(t)}$ can be written as $\left\{\chi_{(t)}^{1}, \chi_{(t)}^{2}, \cdots, \chi_{(t)}^{n}, \cdots, \chi_{(t)}^{d}\right\}$, where $\chi_{(t)}^{n}$ denotes the $n^{\text {th }}$ coordinate of $\chi_{(t)}$.

The signature of a path is a graded infinite series, which contains all the $k^{t h}$ fold iterated integrals. Let $\operatorname{Sig}_{k}(\chi)_{a, b}$ denoted the truncated signature of $\chi$ up to degree $k$ as follows:

$$
\operatorname{Sig}_{k}(\chi)_{a, b}=\left(1, S_{1}(\chi)_{a, b}, S_{2}(\chi)_{a, b}, \cdots, S_{k}(\chi)_{a, b}\right),
$$

with the $k^{t h}$ fold iterated integral calculated as: $S_{k}(\chi)_{a, b}^{n_{1}, n_{2}, \cdots, n_{k}}=\frac{1}{k !} \prod_{j=1}^{k}\left(\chi_{b}^{n_{j}}-\right.$ $\left.\chi_{a}^{n_{j}}\right) . n_{1}, n_{2}, \cdots, n_{k} \in\{1,2, \cdots, d\}$ are the indexes of coordinates.

It is noteworthy that the first fold iterated integral $S_{1}(\chi)_{a, b}$ equals the increment of path $\chi$ during a certain time period. The higher fold iterated integrals and path signature itself have many algebraic and analytic proprieties, which make it an effective feature set of the streamed data. More details about path signature can be found in [2].

Developmental Path Signature. In this work, we construct paths on the growth trajectories of brain regions to explore the geometrical properties of the developmental patterns. For each brain region, their structural feature of 0 year, 1 year and 2 years of age are constructed as a path of length 3 in chronological order. Inspired by [11] that path signature transformation can be integrated into the network as a layer, we propose our developmental path signature as,

$$
P_{i}=\mathbf{W}_{\mathbf{3}} \cdot\left[\operatorname{ReLU}\left(\operatorname{Conv}\left(\mathbf{W}_{\mathbf{1}} \cdot X_{i}\right)\right) \|\left(\mathbf{W}_{\mathbf{2}} \cdot \operatorname{Sig}\left(\mathbf{W}_{\mathbf{1}} \cdot X_{i}\right)\right)\right],
$$


where $\mathbf{W}_{\mathbf{1}}, \mathbf{W}_{\mathbf{2}}$ and $\mathbf{W}_{\mathbf{3}}$ are learnable matrices, which are introduced for a deep integration between path signature theory and machine learning. Sig $g_{k}$ computes the developmental path signature features as defined in equation (2).

\subsection{Inter-region Connectivity Module (ICM)}

Inspired by the fMRI-based connectivity, the relationship between pairs of brain regions may also benefit the sMRI data analyses. Considering the few sampling time points (only 3 here), the Pearson correlation coefficient cannot sufficiently describe the similarity between the growth trajectories of two brain regions. Thereby, with the developmental features extracted, we designed an Inter-region Connectivity Module (ICM) that learns a similarity coefficient for each pair of brain regions, as shown in Fig. 2.

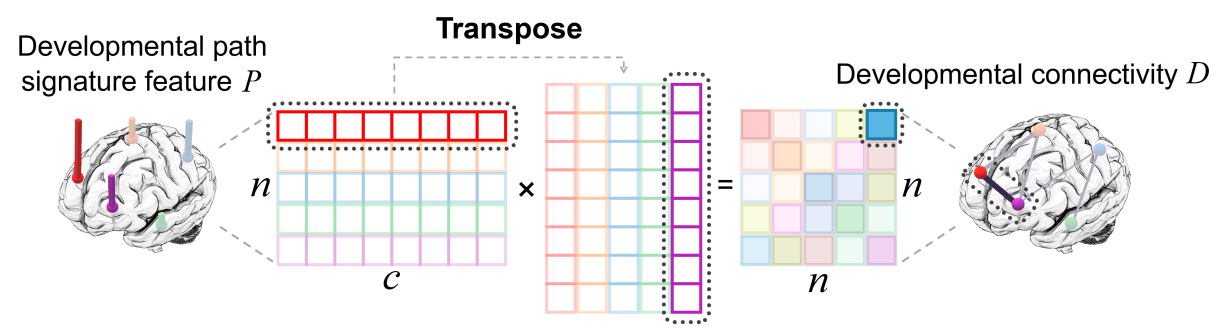

Fig. 2. Illustration of our ICM module. We calculate the cosine similarity of pairs of brain regions by multiplying feature matrices, and then construct a symmetric connectivity matrix $D$. Here $n$ is the number of brain regions and $c$ is the feature dimension.

With the developmental feature $P$ from RDM, we evaluate the connectivity coefficient between the $i^{t h}$ and $j^{t h}$ brain regions as follows:

$$
f_{I C M}\left(P_{i}, P_{j}\right)=\frac{\left(\mathbf{W}_{\mathrm{icm}} \cdot P_{i}\right) \cdot\left(\mathbf{W}_{\mathbf{i c m}} \cdot P_{j}\right)^{\top}}{\left\|\mathbf{W}_{\mathbf{i c m}} \cdot P_{i}\right\|_{2} \cdot\left\|\mathbf{W}_{\mathbf{i c m}} \cdot P_{j}\right\|_{2}} .
$$

Here $P_{i}$ and $P_{j}$ means the developmental feature of region $i$ and $j$. This equation essentially calculates the cosine similarity between the feature vector of $\left(\mathbf{W}_{\mathbf{i c m}} \cdot P_{i}\right)$ and $\left(\mathbf{W}_{\mathbf{i c m}} \cdot P_{j}\right)$. The learnable parameter $\mathbf{W}_{\mathbf{i c m}}$ is introduced to adaptively transform feature for the connectivity evaluation.

By calculating the similarity of all pairs of brain regions, we can get an adjacent matrix $D=\left\{d_{i, j} \mid d_{i, j}=f_{I C M}\left(P_{i}, P_{j}\right), d_{i, j} \in[-1,1]\right\}$. It is based on developmental matrix $P$, so we call $D$ the developmental connectivity. $f_{I C M}\left(P_{i}, P_{j}\right)=$ $f_{I C M}\left(P_{j}, P_{i}\right)$ is obviously symmetric according to equation (4).

\subsection{GCN-based Score Predictor}

Finally, we conduct the multi-layer graph convolution by using developmental connectivity $D \in \mathbb{R}^{70 \times 70}$ in equation (4) as the adjacent matrix , and develop- 
mental features $P \in \mathbb{R}^{70 \times 32}$ in equation (3) as node feature:

$$
P^{l+1}=\operatorname{Re} L U\left(D \cdot P^{l} \cdot \mathbf{W}_{\mathbf{g c n}}+P^{l}\right),
$$

where superscript $l$ means the layer of feature, and $\mathbf{W}_{\mathbf{g c n}}$ is a learnable matrix for GCN predictor. After a two-layer GCN, one fully connected layer is used to predict the cognitive score.

\section{Experiments}

As mentioned in Section 2, we have five cognitive Mullen Scales of early learning, including VRS, FMS, RLS, ELS and ELC. These five tasks are predicted together by the proposed method, and we used Root Mean Squared Error (RMSE) to evaluate the prediction error. L2 loss function is used, and all learnable parameters are initialized randomly and updated by BP algorithm. 5-fold cross validation was taken for comprehensive assessment of the performance.

\subsection{Ablation Study}

In this paper, our CDC-Net consists of two new modules: ICM to adaptively generate inter-region connectivity and RDM to extract the dynamic information of region-wise growth trajectories. Here, we will show the effectiveness of our ICM and developmental path signature by an ablation study.

From the GCN aspect, the connectivity matrix and node features both play important roles. As for connectivity matrix, we compared three types of matrices obtained by Pearson's correlation, graph attention layer (GAT) [22] and our ICM with raw data $\left(A=\left\{a_{i, j} \mid a_{i, j}=f_{I C M}\left(X_{i}, X_{j}\right)\right)\right.$ separately. As for node features, we used the raw feature $X$ in Table 1 . It can be observed that learnable matrices, including GAT and ICM work better than static Pearson's correlation matrix, confirming that our ICM can construct more meaningful brain region connectivity with few time points. Besides, benefited from the explicitly computation in equation (4), our ICM performs better than GAT. In Table 2, we

Table 1. Evaluation of adjacent matrices for GCN backbone.

\begin{tabular}{l||c|c|c|c|c||c}
\hline \multirow{2}{*}{ Methods } & \multicolumn{5}{|c}{ RMSE } \\
\cline { 2 - 7 } & VRS & FMS & RLS & ELS & ELC & Average \\
\hline Pearson & 0.1855 & 0.1951 & 0.1680 & 0.1794 & 0.1757 & $0.1807 \pm 0.0178$ \\
GAT [22] & 0.1784 & 0.1946 & 0.1760 & 0.1771 & 0.1598 & $0.1757 \pm 0.0203$ \\
\hline ICM & $\mathbf{0 . 1 7 2 8}$ & $\mathbf{0 . 1 8 1 9}$ & $\mathbf{0 . 1 4 5 1}$ & $\mathbf{0 . 1 6 4 1}$ & $\mathbf{0 . 1 5 8 6}$ & $\mathbf{0 . 1 6 4 5} \pm \mathbf{0 . 0 1 4 9}$ \\
\hline
\end{tabular}

compared different sequential data descriptor, including GRU [3], LSTM [8] and our Developmental Path Signature (DPS) to serve as the node features. GAT is applied to build the cross-region connectivity. By replacing the other sequential model with our developmental path signature, the overall performance of all five cognitive scores is improved, verifying the effectiveness of our DPS feature. 
Table 2. Evaluation of sequential data descriptor for node features.

\begin{tabular}{l||c|c|c|c|c||c}
\hline \multirow{2}{*}{ Methods } & \multicolumn{6}{|c}{ RMSE } \\
\cline { 2 - 7 } & VRS & FMS & RLS & ELS & ELC & Average \\
\hline LSTM [8] & $\mathbf{0 . 1 7 2 6}$ & 0.1867 & 0.1687 & $\mathbf{0 . 1 7 6 1}$ & 0.1657 & $0.1740 \pm 0.0193$ \\
GRU [3] & 0.1734 & 0.1867 & 0.1687 & 0.1763 & 0.1686 & $0.1717 \pm 0.0228$ \\
\hline DPS & 0.1727 & $\mathbf{0 . 1 8 3 2}$ & $\mathbf{0 . 1 5 0 4}$ & 0.1777 & $\mathbf{0 . 1 5 8 2}$ & $\mathbf{0 . 1 6 8 4} \pm \mathbf{0 . 0 1 9 2}$ \\
\hline
\end{tabular}

\subsection{Comparison with State-of-the-art Methods}

In the past, some machine learning methods have been popular for analyzing the brain sMRI, e.g., KNN [10], SVR [20] and RF [21]. Recently, with the development of deep learning, more effective methods are available, e.g., LSTM [8], GRU [3], GCN [12] and GAT [22].

We compared our CDC-Net with other popular methods mentioned above. Table 3 shows the detailed RMSE of all methods and the ratio of performance improvement compared to KNN. The GCN-based methods perform better than the non-deep learning methods. With the assistance of sequential models to provide developmental information, the performance is further improved. Our proposed CDC-Net performs the best on almost all tasks by introducing the effective inter-region connectivity module and developmental path signature features. Considering the average performance of all tasks, our method reaches a state-of-the-art RMSE 0.1631. It is noteworthy that BrainPSNet[24] introduces the path signature in equation (2) to describe the development pattern of brain regions, but it is poor-performed for neglecting the graph structure among brain regions and the benefits may brought by the learnable matrices in equation (3).

Table 3. Comparison with state-of-the-art methods.

\begin{tabular}{l||c|c|c|c|c||c|c}
\hline \multirow{2}{*}{ Methods } & \multicolumn{7}{|c}{ RMSE } \\
\cline { 2 - 8 } & VRS & FMS & RLS & ELS & ELC & Average & Ratio \\
\hline KNN [10] & 0.1878 & 0.1944 & 0.1680 & 0.1848 & 0.1767 & $0.1823 \pm 0.0220$ & $0 \%$ \\
SVR [20] & 0.1865 & 0.1988 & 0.1692 & 0.1789 & 0.1814 & $0.1830 \pm 0.0216$ & $-0.4 \%$ \\
RF [21] & 0.1892 & 0.1946 & 0.1666 & 0.1801 & 0.1824 & $0.1826 \pm 0.0216$ & $-0.2 \%$ \\
\hline GCN [12] & 0.1855 & 0.1951 & 0.1680 & 0.1794 & 0.1757 & $0.1807 \pm 0.0178$ & $+0.9 \%$ \\
GAT [22] & 0.1784 & 0.1946 & 0.1760 & 0.1771 & 0.1598 & $0.1757 \pm 0.0203$ & $+3.7 \%$ \\
\hline LSTM+GCN & $\mathbf{0 . 1 7 6 5}$ & 0.1805 & 0.1660 & 0.1799 & 0.1644 & $0.1735 \pm 0.0193$ & $+4.8 \%$ \\
GRU+GCN & 0.1805 & 0.1895 & 0.1594 & 0.1683 & 0.1738 & $0.1743 \pm 0.0247$ & $+4.4 \%$ \\
\hline BrainPSNet [24] & 0.1829 & 0.1873 & 0.1587 & 0.1776 & 0.1680 & $0.1749 \pm 0.0179$ & $+4.2 \%$ \\
CDC-Net & 0.1809 & $\mathbf{0 . 1 7 9 6}$ & $\mathbf{0 . 1 4 5 1}$ & $\mathbf{0 . 1 5 6 8}$ & $\mathbf{0 . 1 5 2 9}$ & $\mathbf{0 . 1 6 3 1} \pm \mathbf{0 . 0 1 6 5}$ & $+\mathbf{1 1 . 5 \%}$ \\
\hline
\end{tabular}

\subsection{Illustration of Brain Region Developmental Connectivity}

By averaging the developmental connectivity matrices of all subjects, we can get the general brain region developmental connectivity, shown in Fig. 3. Fig. 3(a) 
is the whole connectivity matrix, while Fig. 3(b) only shows the top $3 \%$ strong connections. Our 70 brain regions can be divided into 5 larger areas on each side of the brain, and details can be consulted in [4].

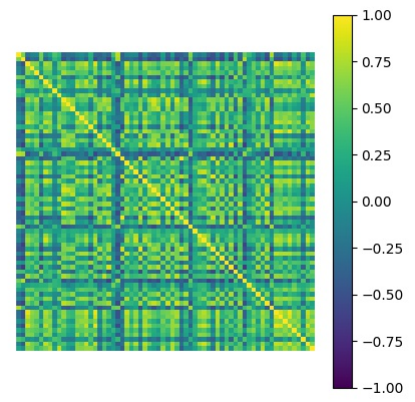

(a)

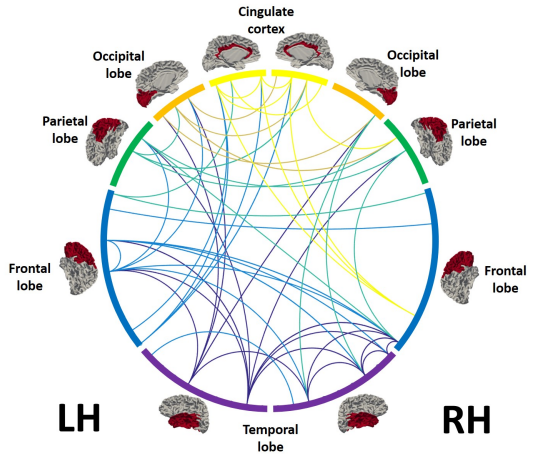

(b)

Fig. 3. Illustration of Brain Region Developmental Connectivity. (a) shows the whole connectivity matrix and (b) shows top $3 \%$ strong connections.

Fig. 3(a) shows a phenomenon of modularization, indicating that brain regions in the same area have similar function. Considering the top $3 \%$ connections in Fig. 3(b), most of the connections are concentrated in temporal lobe and frontal lobe. Temporal lobe plays an important role in organizing language, while frontal lobe controls the execution of voluntary of muscle movement and other high-order functions. This is consistent with Mullen Scales assessment which mainly involves language and motor skills. The brain regions with the strongest connections incluce the precuneus cortex, transverse temporal cortex and cuneus cortex, which are all involved in vision or language.

\section{Conclusion}

In the task of infant cognitive score prediction based on longitudinal brain structure data, we proposed two novel modules: Region-wise Developmental Module $(\mathrm{RDM})$ and Inter-region Connectivity Module (ICM). RDM is the feature extractor to explore the developmental information of brain regions, and ICM is the connectivity matrix builder to construct connections between brain regions. Our proposed method with RDM, ICM and 2-layer GCN backbone obtains the state-of-the-art prediction result. Furthermore, by visualizing the brain region developmental connectivity learned by RDM and ICM, we find that several brain regions associated with cognitive ability are connected, demonstrating the rationality of our proposed framework. 


\section{References}

1. Adeli, E., Meng, Y., Li, G., et al.: Multi-task prediction of infant cognitive scores from longitudinal incomplete neuroimaging data. NeuroImage 185, 783-792 (2019)

2. Chevyrev, I., Kormilitzin, A.: A primer on the signature method in machine learning. arXiv preprint arXiv:1603.03788 (2016)

3. Cho, K., Van Merriënboer, B., Gulcehre, C., et al.: Learning phrase representations using rnn encoder-decoder for statistical machine translation. arXiv preprint arXiv:1406.1078 (2014)

4. Desikan, R.S., Ségonne, F., Fischl, B., et al.: An automated labeling system for subdividing the human cerebral cortex on mri scans into gyral based regions of interest. NeuroImage 31(3), 968-980 (2006)

5. Fischl, B., Sereno, M.I., Dale, A.M.: Cortical surface-based analysis: Ii: inflation, flattening, and a surface-based coordinate system. NeuroImage 9(2), 195-207 (1999)

6. Ghribi, O., Li, G., Lin, W., et al: Multi-regression based supervised sample selection for predicting baby connectome evolution trajectory from neonatal timepoint. Medical Image Analysis 68, 101853 (2021)

7. Griffanti, L., Rolinski, M., Szewczyk-Krolikowski, K., et al.: Challenges in the reproducibility of clinical studies with resting state fmri: An example in early parkinson's disease. NeuroImage 124, 704-713 (2016)

8. Hochreiter, S., Schmidhuber, J.: Long short-term memory. Neural computation $\mathbf{9}(8), 1735-1780(1997)$

9. Kagan, J., Herschkowitz, N.: A young mind in a growing brain. Psychology Press (2006)

10. Keller, J.M., Gray, M.R., Givens, J.A.: A fuzzy k-nearest neighbor algorithm. IEEE Transactions on Systems, Man, and Cybernetics SMC-15(4), 580-585 (1985)

11. Kidger, P., Bonnier, P., Perez Arribas, I., et al.: Deep signature transforms. Advances in Neural Information Processing Systems 32, 3105-3115 (2019)

12. Kipf, T.N., Welling, M.: Semi-supervised classification with graph convolutional networks. In: Proceedings of International Conference on Learning Representations (2017)

13. Lai, S., Zhu, Y., Jin, L.: Encoding pathlet and sift features with bagged vlad for historical writer identification. IEEE Transactions on Information Forensics and Security 15, 3553-3566 (2020)

14. Li, C., Zhang, X., Liao, L., et al.: Skeleton-based gesture recognition using several fully connected layers with path signature features and temporal transformer module. In: Proceedings of the AAAI Conference on Artificial Intelligence. pp. 8585-8593 (2019)

15. Li, G., Nie, J., Wu, G., et al.: Consistent reconstruction of cortical surfaces from longitudinal brain mr images. NeuroImage 59(4), 3805-3820 (2012)

16. Li, G., Wang, L., Shi, F., et al.: Mapping longitudinal development of local cortical gyrification in infants from birth to 2 years of age. Journal of Neuroscience 34(12), 4228-4238 (2014)

17. Liao, L., Zhang, X., Li, C.: Multi-path convolutional neural network based on rectangular kernel with path signature features for gesture recognition. In: Proceedings of IEEE Visual Communications and Image Processing. IEEE (2019)

18. Seidlitz, J., Váša, F., Shinn, M., et al: Morphometric similarity networks detect microscale cortical organization and predict inter-individual cognitive variation. Neuron 97(1), 231-247 (2018) 
19. Smith, S.M., Vidaurre, D., Beckmann, C.F., et al.: Functional connectomics from resting-state fmri. Trends in Cognitive Sciences 17(12), 666-682 (2013)

20. Smola, A.J., Schölkopf, B.: A tutorial on support vector regression. Statistics and Computing 14(3), 199-222 (2004)

21. Svetnik, V., Liaw, A., Tong, C., et al.: Random forest: a classification and regression tool for compound classification and qsar modeling. Journal of Chemical Information and Computer Sciences 43(6), 1947-1958 (2003)

22. Veličković, P., Cucurull, G., Casanova, A., et al.: Graph attention networks. In: Proceedings of International Conference on Learning Representations (2018)

23. Zhang, C., Adeli, E., Wu, Z., et al.: Infant brain development prediction with latent partial multi-view representation learning. IEEE Transactions on Medical Imaging 38(4), 909-918 (2018)

24. Zhang, X., Cheng, J., Ni, H., et al.: Infant cognitive scores prediction with multistream attention-based temporal path signature features. In: Proceedings of International Conference on Medical Image Computing and Computer-Assisted Intervention (MICCAI). Springer (2020)

25. Zhao, T., Xu, Y., He, Y.: Graph theoretical modeling of baby brain networks. NeuroImage 185, 711-727 (2019) 